\title{
Pathways, processes, team work: paving the way for value-based care with the quadruple aim
}

\author{
Linda D Bosserman MD, FACP
}

W hile we can imagine what a high-quality, stateof-the-art cancer care system might deliver in terms of value-based care and how it might yield exceptional patient outcomes and job satisfaction for our staff, most of us are struggling with the processes and tools needed to achieve defined outcomes that can be benchmarked and further refined - all within the limits of our already hectic 12-, 14,- 16+-hour days. As more community practices, academic centers, and hospitals align to share and leverage expertise in their efforts to form more streamlined, patient-centered delivery systems for cancer care, we need to set up, refine, and integrate pathways into pathway programs that will pave the way to the delivery of value-based care. The key is in implementation approaches and empowering technology, so that the teambased, patient-centered work can be done within a normal workday. These approaches could serve to reverse the growing burden of burnout among oncology professionals while meeting the expanded demands of oncology care.

\section{The case for pathways}

As our understanding of individual cancers and their unique patient milieus deepens, we need validated, evidence-based pathways for developing standard and modifiable individual treatment plans with real-time decision-making options integrated into the points of care to effectively deliver quality cancer care with reportable clinical and financial outcomes. Pathways were initially rejected as "cook books" but have been shown to improve care and often lower costs. ${ }^{1,2}$ Given the explosion of subtypes of tumor pathologies and molecular mutations, as well as the refining of staging and recognition of the importance of risk factors and comorbidities that all determine what care will provide the best outcomes, even doctors who treat only one cancer can benefit from coming together to standardize approaches for patients. Pathways also offer reassurance to patients that their care plans reflect the integration of their health preferences; diagnostics; as well as their initial, follow-up, and sequential therapies based on the current evidence of benefit with specified toxicities for the best price. If patients have a better understanding of and engagement in their care plans, we can hope for greater compliance and better, earlier management of toxicities to lessen their suffering. In addition, pathways can be great tools for sharing evidence-based written information with patients about the myriad details discussed during consultations, which are often held in times of significant stress for patients and during which learning processes and memory function are impaired. ${ }^{3}$ Pathways also provide individualized and evidence-based decision tools that can help counter the many different sources of information patients turn to in trying to ensure their treatment choices are the best possible for them. They can also support expanded clinical trial participation, which in the age of targeted and customized immune-based therapies holds the greatest promise for increasing cure rates.

\section{The need for pathways programs}

Beyond practices adopting pathways for therapy and supportive care, is the need to develop pathway programs that integrate the pathways into the care process through electronic medical records (EMRs) for physician and patient education, documentation, payment authorizations, and standardized ordering, as well as tracking patients' outcomes and providing them with their individual metrics. Given the limits of data from randomized clinical trials, which engage fewer than $5 \%$ of patients and underrepresent the diversity of the patients we see in regard to age, geographic location, tumor diversity, genetic inheritance, and comorbidities, collecting structured data on pathway use and outcomes for our diverse patient population could help us further personalize therapies and minimize toxicities. Data-sharing systems such as CancerLinq that harness real-time data from millions of cancer patient records 
will enable comparisons of patient data with the data from other patients like them and enhance the role of clinicians as care partners with patients. Together they can weigh the diagnostic and therapeutic options to arrive at a truly personalized care plan. Using such sophisticated and real-time databases could also expand access to clinical trials and serve as outcome registries for patients in the United States and globally. We can then learn more completely how to reduce the burden of cancer.

\section{The human factors: attention and distraction}

A presentation on human factor research by Rachael Gilbert at the 2016 ASCO Survivorship Conference in San Francisco provided eye-opening data on the limits of human attention, pointing out that our attention is finite and not expandable. ${ }^{4}$ She reviewed how damaging frequent distractions are and how overwhelming demands on human attention lead to errors and poor outcomes. This is something the airline industry has long been tuned in to, and which medical leaders need to embrace. As we move to standardize processes and pathways for therapy and supportive care, improve patient engagement, and integrate clinical research into our care systems, we have to agree that oncology physicians can then oversee those steps, without personally executing each step. We need to pilot and establish optimal teams working under physicians to order and document the desired and delivered care. Human factor research informs us about the importance of highly trained staff being able to focus fully on the tasks that require their training and expertise while minimizing wasted time and distractions. Requiring highly trained clinicians to focus on "clicks for compliance" within in the EMR is not only poor use of their expertise, it distracts and often prevents them from their most important tasks: thinking about their patients, engaging with them in a comprehensive care plan throughout their care continuum, and contributing to the scientific advances within oncology .

The $40 \%-50 \%$ of the workday that clinicians spend on nonproductive work is a major contributor to the growing burnout rates that Medscape's 2016 survey reports as 46\% for oncologists ${ }^{5}$ (58\% of female and $40 \%$ of male oncologists). The survey also found that only $29 \%$ of oncologists were very or extremely happy at work. The main contributors to burnout and dissatisfaction were too many bureaucratic tasks, too many hours at work, increased computerization, and maintenance of certification requirements, all issues that have been made worse by expanding care demands, inefficient tools, and lack of team-based care, with physicians being expected to "click for compliance" in person, and therefore spend more time at work and away from their patients.

From early in medical school, clinicians and other medical staff grapple with the fact that they won't remember everything they have learned, won't read every relevant journal article, won't be able to meet every need for every patient and every compliance requirement... Clinicians are constantly weighing priorities and making choices about how to allot their time. Most experienced clinicians find a balance they can live with. Many have built teams and piloted team-based care, but there has never been enough time to do it all and have any balance in personal lives. Now, with the explosion of trackable ordering and the need for more and more detailed discrete patient data, most compliance officers are requiring doctors enter every click. Thus, despite the benefits of technology, doctors are even more overburdened - and yes, distracted - by having to feed the EMR beast. We need process engineers, technology innovations, and thoughtful clinicians to improve EMR and team-based care processes with physician signoff if we have any hope of addressing burnout and workforce shortages and improving job satisfaction. These critical and growing issues of burnout and shortages in the oncology workforce threaten our ability to have an energized, empowered, and engaged workforce to serve our patients and ourselves. Integrated team-based care then offers the real promise of a brighter future.

\section{Team-based care and new compliance standards}

In recent years, the American Medical Association (AMA), the American Society of Clinical Oncology (ASCO) and the National Cancer Institute (NCI) have taken steps to address the growing workloads and rates of burnout among health care professionals, including oncologists and oncology midlevel providers. A systematic review of team-based care by Taplin and colleagues noted that there is evidence from other fields to suggest that "a team-based approach helps organize and optimize tasks that involve individuals and groups, but team effectiveness has not been fully evalu-

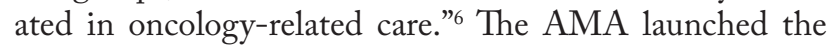
StepsForward program, which outlines strategies for teambased care and for physicians to recharge their practices by targeting work-related stress and improving job satisfaction among staff. ${ }^{7}$ ASCO has also worked to develop programs and has offered presentations at its annual meetings to promote physician wellness and prevent burnout. An ASCO task force is working to define the components of physician workload for physician payment reform, and the Task Force on Clinical Pathways has developed a policy statement that includes their efficient implementation as a core principle. ${ }^{8}$

The need for developing effective team-based care in cancer led ASCO and the NCI to develop a working group that held a workshop in February 2016. The purpose of the Teams in Cancer Care Delivery Initiative is to bring the 2 groups together "to learn about current research in team-based cancer care and explore changes that that will positively affect team-based care for patients with can- 
cer." Several groups presented their initial pilot work at the ASCO-NCI team meeting, and the presentations are being readied for publication later this year. We can look forward to learning from these pilots how to best implement team-based care to deliver high-quality cancer care and execute meaningful clinical research with patient and provider satisfaction that meets the quadruple aim. ${ }^{10}$

But teams need to have flexibility in completing tasks overseen by physicians. Before the advent of EMRs, trained office staff helped clinicians meet patient care needs and place orders. Doctors would come out of busy exam rooms and ask trained medical staff to draw specific labs, or do an ECG, schedule a consult or a follow-up visit, call for prescription refills, or call in prescriptions for a practice's standard regimen, say, for nausea or diarrhea or constipation. They later noted the plans in their notes. In the new era of EMR tracking, however, busy doctors are now told to stop (which usually means disengaging with one's clinical work and the patient), go the EMR, sign on, find the patient file in the system, find the order sections, and then click on each order before anything can be done. As practices have standardized therapy and supportive care regimens, doctors should be able to note or give verbal orders for standard therapies, symptom control packages, and follow-up under clearly defined practice policies and staff licensure requirements. The appropriate staff should then be able to enter the clicks, which a doctor would later authorize in batches so that his or her attention remains focused on the patient.

Doctors' attention is actually most critical to nonstandard care needs: ensuring decisions on warranted variations and determining when a set of symptoms or tests is not providing a complete picture to make a final care plan recommendation and explaining clinical trial options. The current emphasis on detailed, time-consuming, routine discrete documentation in the EMR is robbing clinicians of their attention and time to focus on the most important elements of their work - their patients' individual care plans, participation in clinical trials, and keeping current with new developments - and it fuels the rising burnout rates. If we are to meet the growing need to think more critically about every patient, we need to value thinking time, recognize the limits of human attention, and challenge the current volume-based compliance standards to allow pilot tests and best practices to emerge to facilitate the growing workload by staff teams led by physicians.

\section{The case for patient engagement}

From the patient perspective, more engagement and shared decision making requires appropriate educational interactions and real-time symptom management and triage to keep patients as healthy as possible during their complex therapies. It means educating patients about the array of symptoms, some of which we can fix or which they will have to tolerate, and others will need to be treated as soon as they emerge both to relieve suffering and prevent worsening that could lead to emergency room visits and hospitalizations. Given these complexities, an important component in value-based care is the development of effective patient-reported outcome (PRO) tools that prompt patients to report their symptoms with the use of a growing array of triage management tools such as EMR portals, web-based applications, and phone apps that alert staff to toxicities. Staff can then proactively contact patients and oversee changes in their home care or direct them to the clinic or the emergency department.

Keeping patients as healthy as possible by minimizing toxicities and treating symptoms as they emerge can reduce the number of hospital and emergency department visits, but it requires enhanced engagement between the patient and caregiver teams. In addition, this engagement includes better initial educational tools on treatment plan choices and does not stop when therapy finishes. Many patients have lingering recurrence anxieties or residual treatmentrelated toxicities that need management to avoid unnecessary testing and achieve optimum patient health and wellbeing. As the number of cancer survivors and patients with cancer as a chronic illness increases, serving their physical, social, and emotional needs is a key challenge to achieving ongoing value-based cancer care that new processes, team work, and technology can address.

\section{Plan of action}

Validated, evidence-based pathways need to be adopted and integrated into point of service technology that facilitates education, warranted variations, and clinical trials. Patients need to be fully engaged in shared decision making and real-time symptom management. Our professional societies - ASCO, AMA, the American Society of Hematology, the NCI - along with the Centers for Medicare and Medicaid Services and our payers need to expedite pilots that promote team-based, physician-led, accountable cancer care that divides up the workload to achieve accessible, affordable patient-centered care.

We need to figure out the best use of specially trained teams of clerical staff, scribes, medical assistants, licensed vocational nurses, registered nurses, oncology certified nurses, therapists, social workers, pharmacists, and advanced practice providers, all led by physicians. ${ }^{11} \mathrm{We}$ need to develop defined work goals, care processes, and discrete data needs for clinical, financial, and quality assessments. Once those parameters have been defined, we need to develop EMRs and PRO tools to efficiently prompt, collect, and integrate relevant, real-time data from patients, clinicial teams, and other providers.

Value-based care is within our capabilities, but not unless we embrace evidence-based pathways, integrate technol- 


\section{From the Editor}

ogy support, and allow team-based division of the workload, led by experienced physicians. Care delivery can then further benefit from ongoing refinements informed by real-

\section{References}

1. Neubauer MA, Hoverman JR, Kolodziej M, et al. Cost effectiveness of evidence-based treatment guidelines for the treatment of non-small-cell lung cancer in the community setting. J Oncol Pract. 2010:6:12-18.

2. Hoverman JR, Cartwright TH, Patt DA, et al. Pathways, outcomes, and costs in colon cancer: retrospective evaluations in two distinct databases. J Oncol Pract. 2011;7(3 suppl):52s-59s.

3. Derakshan N, Eysenck MW. Anxiety, processing efficiency, andcognitive performance. New developments from attentional control theory. Eur Psychol. 2009;14:168-176.

4. Gilbert R. The human factor: designing safety into oncology practice. http://meetinglibrary.asco.org/content/119279?media=sl. Presentation at ASCO Quality Care Symposium, Phoenix, AZ, February 27, 2016. Accessed June 22, 2016.

5. Peckham C. Medscape lifestyle report 2016: bias and burnout. http:// www.medscape.com/features/slideshow/lifestyle/2016/public/overview. Released January 13, 2016. Accessed June 22, 2016. time scientific, personal, clinical, and financial outcomes to achieve true value-based care by a highly trained, vibrant, and satisfied workforce.

6. Taplin SH, Weaver S, Salas E, et al. Reviewing cancer care team effectiveness. J Oncol Pract. 2015;11:239-246.

7. American Medical Association. STEPSforward program. https:// www.stepsforward.org/. Launched June 2015. Accessed June 22, 2016.

8. Zon RT, Frame JN, Neuss MN, et al. American Society of Clinical Oncology policy statement on clinical pathways in oncology. J Oncol Practice. 2016:12;261-266.

9. Lambert J. NCI-ASCO partner for Teams in Cancer Care Delivery workshop. https://am.asco.org/nci-asco-partner-teams-cancer-caredelivery-workshop. Published May 26, 2016. Accessed June 19, 2016.

10. Bodenheimer T, Sinsky C. From triple aim to quadruple aim: care of the patient requires care of the provider. Ann Fam Med. 2014; 12:573-576.

11. Spiegelman P, Berrett B. Patients come second: leading change by changing the way you lead. Austin, Texas: Greenleaf Book Group; 2013. 